\title{
Diallyl thiosulfinate enhanced the anti- cancer activity of dexamethasone in the side population cells of multiple myeloma by promoting miR-127-3p and deactivating the PI3K/AKT signaling pathway
}

\author{
Wenfeng $\mathrm{He}^{1+}$, Yonghui $\mathrm{Fu}^{2+}$, Yongliang Zheng ${ }^{3}$, Xiaoping Wang ${ }^{4}$, Bin $\mathrm{Liu}^{3}$ and Junquan Zeng ${ }^{3,5^{*}}$ (I)
}

\begin{abstract}
Background: Side population (SP) cells, which have similar features to those of cancer stem cells, show resistance to dexamethasone (Dex) treatment. Thus, new drugs that can be used in combination with Dex to reduce the population of SP cells in multiple myeloma (MM) are required. Diallyl thiosulfinate (DATS, allicin), a natural organosulfur compound derived from garlic, has been shown to inhibit the proliferation of SP cells in MM cell lines. Therefore, we investigated the effect of a combination of DATS and Dex (DAT + Dex) on MM SP cells.

Methods: SP cells were sorted from MM RPMI-8226 and NCI-H929 cell lines using Hoechst 33342-labeled fluorescence-activated cell sorting. The growth of SP cells was evaluated using the cell counting kit-8 assay. Cell cycle and apoptosis assays were conducted using a BD Calibur flow cytometer. miRNA expression was measured using quantitative reverse transcription-polymerase chain reaction. Phosphoinositide 3-kinase (PI3K), phosphorylated AKT (p-AKT), AKT, p-mechanistic target of rapamycin (mTOR), and mTOR levels were measured using western blot analysis.

Results: Our results showed that the combination of DATS+Dex inhibited sphere formation, colony formation, and proliferation of MM SP cells by inducing apoptosis and cell cycle arrest in the G1/S phase. In addition, the combination of DATS+Dex promoted miR-127-3p expression and inhibited PI3K, p-AKT, and p-mTOR expression in SP cells. Knockdown of miR-127-3p expression weakened the effect of DATS+Dex on cell proliferation, colony formation, apoptosis, and cell cycle of MM SP cells. Additionally, knockdown of miR-127-3p activated the PI3K/AKT/ mTOR signaling pathway in MM SP cells cotreated with DATS+Dex.

(Continued on next page)
\end{abstract}

\footnotetext{
*Correspondence: junquan_zeng@126.com

${ }^{+}$Wenfeng He and Yonghui Fu contributed equally to this work.

${ }^{3}$ Department of Hematology, The Affiliated Hospital of Jinggangshan University, ji'an 343000, Jiangxi Province, China

${ }^{5}$ Department of internal medicine, Jinggangshan University, ji'an 343009, Jiangxi Province, China

Full list of author information is available at the end of the article
}

(C) The Author(s). 2021 Open Access This article is licensed under a Creative Commons Attribution 4.0 International License, which permits use, sharing, adaptation, distribution and reproduction in any medium or format, as long as you give appropriate credit to the original author(s) and the source, provide a link to the Creative Commons licence, and indicate if changes were made. The images or other third party material in this article are included in the article's Creative Commons licence, unless indicated otherwise in a credit line to the material. If material is not included in the article's Creative Commons licence and your intended use is not permitted by statutory regulation or exceeds the permitted use, you will need to obtain permission directly from the copyright holder. To view a copy of this licence, visit http://creativecommons.org/licenses/by/4.0/ The Creative Commons Public Domain Dedication waiver (http://creativecommons.org/publicdomain/zero/1.0/) applies to the data made available in this article, unless otherwise stated in a credit line to the data. 
(Continued from previous page)

Conclusion: We demonstrated that cotreatment with DATS+Dex reduced cell proliferation, promoted apoptosis, and caused cell cycle arrest of MM SP cells by promoting miR-127-3p expression and deactivating the PI3K/AKT/ mTOR signaling pathway.

Keywords: Multiple myeloma, Side population cells, Diallyl trisulfide, Dexamethasone, miR-127-3p, PI3K signaling pathway

\section{Background}

Multiple myeloma (MM) is a type of cancer that causes abnormal proliferation of plasma cells in the bone marrow [1]. Despite considerable progress in the treatment of MM, the 5-year survival rate of patients with this cancer is only approximately $47 \%$, mainly because of the prevalence of drug resistance and tumor relapse [2], which are associated with the existence of cancer stem cells (CSCs). Side population (SP) cells have many similar features to those of CSCs and can be separated from main population (MP) cells using flow cytometry-based sorting with Hoechst 33342 staining [3]. SP cells have important pathophysiological and clinical characteristics that strongly correlate with tumor relapse and drug resistance [4]. Dexamethasone (Dex) is a drug commonly used for the treatment of MM [5], but SP cells show resistance to Dex treatment [6].

Thus, there is a need to discover new safer drugs that can be used in combination with Dex to reduce SP cell population in MM. Diallyl thiosulfinate (DATS, allicin) is a natural organosulfur compound derived from garlic that has been investigated as potential anticancer drug $[7,8]$. DATS inhibits proliferation and promotes apoptosis of SP cells in MM [9]. Additionally, several studies have shown that DATS-induced apoptosis involves the phosphoinositide 3-kinase (PI3K)/AKT/mechanistic target of rapamycin (mTOR) signaling pathway [10-12]. However, whether DATS can enhance the inhibitory effect of Dex on the proliferation of SP cells remains unclear.

MicroRNAs (miRNAs), small noncoding RNA molecules that modulate gene expression, have been confirmed to have prognostic significance in MM [13]. miRNAs also regulate MM growth, metastasis, and chemotherapy sensitivity by inhibiting target gene expression [14-16]. A previous study found that miR-451 promoted the proliferation and colony formation of SP cells in MM [17]. However, whether miRNAs could be beneficial as a cotreatment with DATS and Dex (DATS+ Dex) in regulating SP cell proliferation remains unclear.

In this study, we investigated the effects of cotreatment with DATS+Dex on the proliferation, cell cycle, and apoptosis of MM SP cells. Subsequently, we analyzed the potential role of miRNA and the PI3K/AKT/mTOR signaling pathway in the regulatory mechanism involved in the effect of this combination on MM SP cells.

\section{Methods}

Cell culture, SP cell separation, and treatments

The human MM RPMI-8226 (CCL-155) and NCI-H929 (CRL-9068) cell lines were obtained from American Type Culture Collection (Manassas, VA, USA) and cultured as previously described [18]. SP cells were sorted from the MM RPMI-8226 and NCI-H929 cell lines using Hoechst 33342-labeled fluorescence-activated cell sorting as previously described [18]. SP cells were separately treated with $10 \mu \mathrm{g} / \mathrm{mL}$ DATS [9], $50 \mu \mathrm{M}$ Dex (Sigma Aldrich, St Louis, MO, USA) [6], and cotreatment with $10 \mu \mathrm{g} / \mathrm{mL}$ DATS $+50 \mu \mathrm{M}$ Dex, and untreated cells were used as a control group.

\section{Proliferation, colony formation, and sphere formation assays}

The growth of RPMI-8226 and NCI-H929 cells was evaluated using the MTS (Beyotime, Shanghai, China) in accordance with the manufacturer's instructions. A colony formation assay was performed as previously described [19]. For sphere formation assays, SP cells were seeded in ultra-low attachment plates under stem cell conditions by culturing in Dulbecco's modified Eagle's medium-F12 culture medium containing $20 \mathrm{ng} / \mathrm{mL}$ epidermal growth factor, $10 \mathrm{ng} / \mathrm{mL}$ basic fibroblast growth factor, and 1× B-27 supplement, under saturated humidity conditions at $37{ }^{\circ} \mathrm{C}$ with $5 \% \mathrm{CO}_{2}$. The spheres were visualized using microscopy (Olympus, Tokyo, Japan).

\section{Cell cycle and apoptosis analysis}

Cell cycle and apoptosis assays were conducted using the Cell Cycle Detection and apoptosis detection kits (Keygen, Nanjing, China), respectively. The samples were analyzed using a BD Calibur flow cytometer (BD, Franklin Lakes, NJ, USA).

\section{Western blot analysis}

Total protein isolation, concentration determination, and western blotting were performed as previously described [18]. Protein was extracted from SP cells and determined using a protein assay kit (Keygentec, Nanjing, China). Denatured protein samples $(20 \mu \mathrm{g})$ were separated using $10 \%$ sodium dodecyl sulfate-polyacrylamide gel electrophoresis and transferred onto polyvinylidene fluoride membranes (Millipore, Billerica, MA, USA). 
Table 1 Primer sequence

\begin{tabular}{lll}
\hline Gene & Primer sequence (5'-3') & Size (bp) \\
\hline miR-138 F & ACACTCCAGCTGGGAGCTGGTGTTGGAATC & 73 \\
miR-138 R & CTCAACTGGTGTCGTGGA & \\
miR-3200-5p F & ACACTCCAGCTGGGATCTGAGAAGGCGCA & 72 \\
miR-3200-5p R & CTCAACTGGTGTCGTGGA & \\
miR-127-3p F & ACACTCCAGCTGGGTCGGATCCGTCTGAGC & 72 \\
miR-127-3p R & CTCAACTGGTGTCGTGGA & \\
U6F & CTCGCTTCGGCAGCACA & 94 \\
U6 R & AACGCTTCACGAATTGCGT & \\
ALDH1 F & TCACAGGATCAACAGAGGTTGG & \\
ALDH1 R & GCCCTGGTGGTAGAATACCC & 231 \\
Sox2 F & TACAGCATGATGCAGGACCA & 138 \\
Sox2 R & CTCGGACTTGACCACCGAAC & \\
GAPDH F & GCTCATTTGCAGGGGGGAG & \\
GAPDH R & GTTGGTGGTGCAGGAGGCA &
\end{tabular}

ALDH1 Aldehyde dehydrogenase 1, Sox2 Sex determining region $Y$ (SRY)-box 2, GAPDH Glyceraldehyde 3-phosphate dehydrogenase, $F$ Forward, $R$ Reverse
After blocking, the membranes were incubated at $4{ }^{\circ} \mathrm{C}$ overnight with primary antibodies. Then, the membranes were washed and incubated with horseradish peroxidase (HRP)-conjugated goat anti-IgG H\&L secondary antibodies $(1: 20,000)$ for $2 \mathrm{~h}$ at $25^{\circ} \mathrm{C}$. The protein bands were visualized using an enhanced chemiluminescence (ECL) kit (Thermo Scientific, Rockford, IL, USA) and quantified using the Image Lab 6.0 software. The expression of all proteins was normalized to that of glyceraldehyde 3-phosphate dehydrogenase. The blots were incubated with the following primary antibodies: antiPI3K (1:500), anti-p-AKT (1:500), anti-total AKT (tAKT, 1:200), anti-mTOR antibody (1:1000), and anti-pmTOR antibody (1:500) (all from Santa Cruz).

GEO data analysis and quantitative reverse transcriptionpolymerase chain reaction (qRT-PCR)

miRNA expression datasets (GSE56163) were downloaded from Gene Expression Omnibus (http://www.ncbi.nlm. nih.gov/geo), a high-throughput gene expression database, and analyzed using GEO2R analysis. miRNA datasets of SP and MP cells in MM were compiled using the

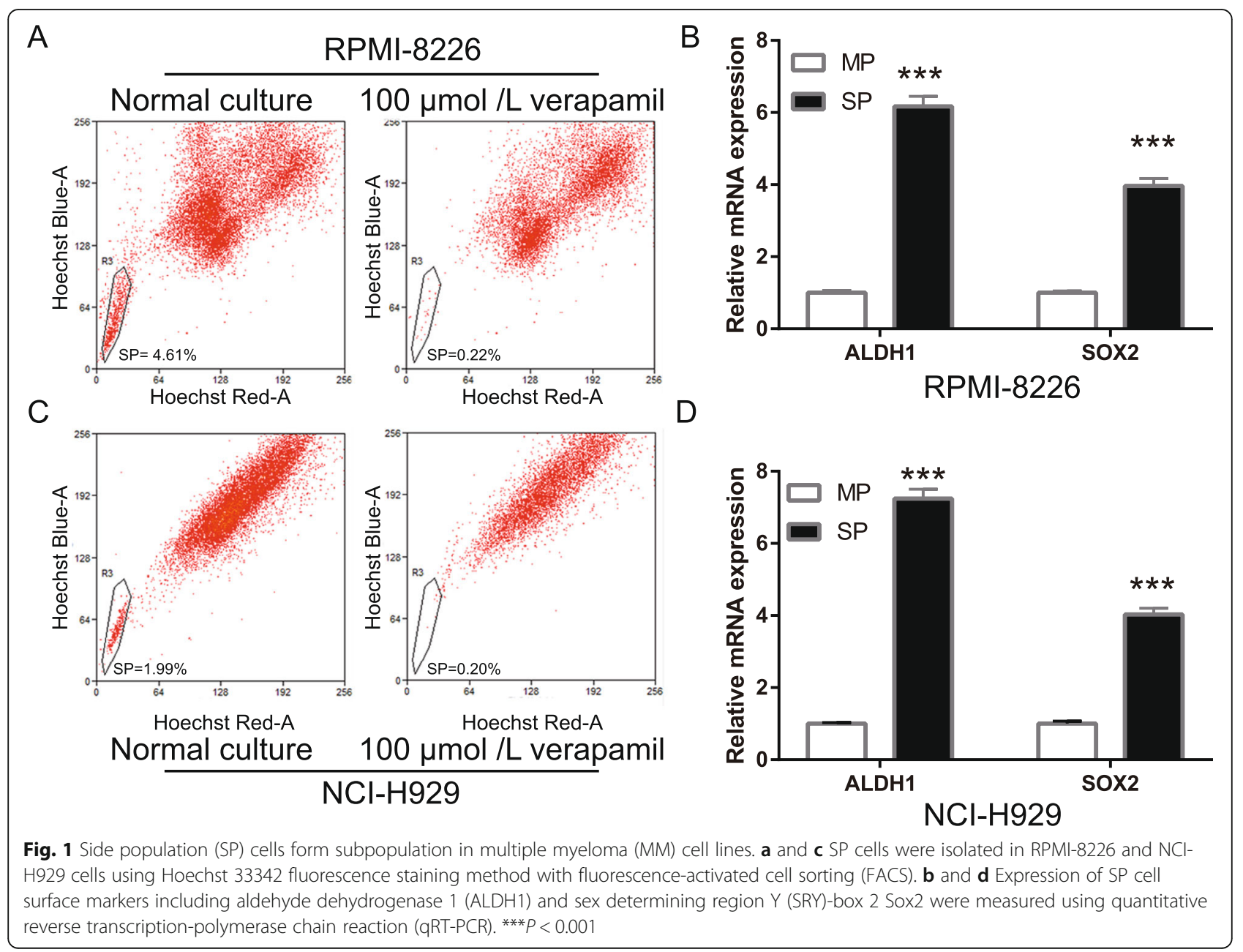


GPL11434 miRCURY LNA microRNA Array, 6th generation, and were analyzed using qRT-PCR. Briefly, SP cells were collected, followed by extraction of total RNA using TRIzol reagent (Invitrogen) and reverse transcription to complementary DNA using an ImProm-II reverse transcription system (Promega). The expression of miRNAs and mRNA was determined using SYBR GREEN qPCR Super Mix (Invitrogen) with the U6 gene as the internal reference. All experiments were performed in duplicate and repeated three times. The results are represented as fold-induction, which was calculated using the $2^{-\Delta \Delta C T}$ method. The primers used to determine the expression of miRNAs are shown in Table 1.

\section{Statistical analyses}

All statistical analyses were performed using statistical package for the social sciences version 19.0. (IBM Inc., USA). Continuous variables are presented as means \pm standard deviations. The differences between multiple groups were analyzed using a one-way analysis of variance, followed by a post-hoc least significance difference test. An independent $t$-test was used to compare differences between groups, and two-sided $P$-values $<0.05$ were considered statistically significant.

\section{Results}

SP cells formed subpopulation in MM cell lines

To investigate the effect of cotreatment with DATS+Dex on SP cells in MM, SP cells were isolated from RPMI-8226 and NCI-H929 cells using the Hoechst 33342 fluorescence-activated cell sorting. The results showed that the number of SP cells in RPMI-8226 and NCI-H929 cells was $4.65 \pm 0.125$ and $1.99 \pm 0.138$, respectively (Fig. $1 \mathrm{a}$ and c). Then, the expression of the SP cell surface markers,

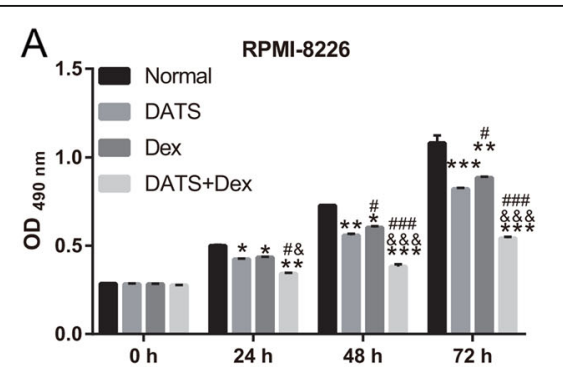

B
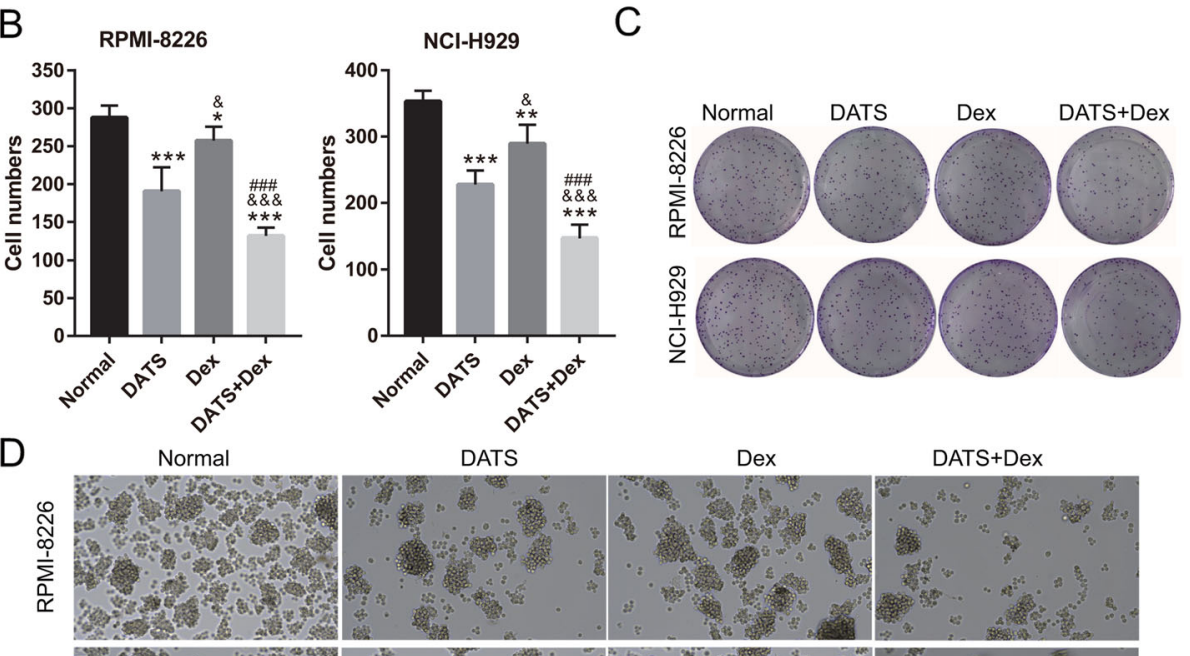

N
$\frac{1}{1}$
$\frac{1}{U}$
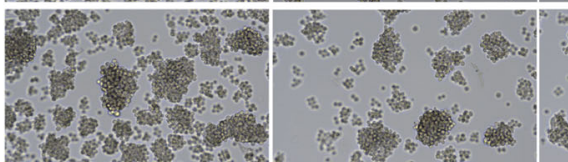

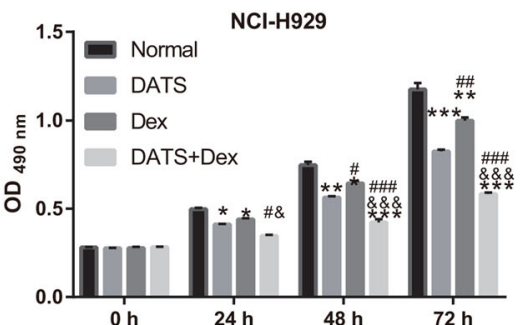

C

Fig. 2 Diallyl thiosulfinate and dexamethasone (DATS+Dex) cotreatment inhibited proliferation and colony formation of multiple myeloma (MM) side population (SP) cells. a Effect of DATS, Dex, and DATS+Dex treatments on proliferation of MM SP cells detected using MTS analysis at 0, 24, 48, $72 \mathrm{~h}$ after treatment. $\mathbf{b}$ Bar represents cell numbers of colonies formed in SP cells after treatment with DATS, Dex, or DATS+Dex. $\mathbf{c}$ Representative image of colony formation by SP cells after treatment with DATS, Dex, or DATS+Dex. $\mathbf{d}$ Representative image of spheroids formation by SP cells after treatment with DATS, Dex, or DATS+Dex (magnification, $\times 100$ ). ${ }^{* * *} P<0.001$ vs normal group, ${ }^{\&} P<0.05$ and ${ }^{\& \& \&} P<$ 0.001 vs DATS group, and ${ }^{\# \# \# P} P 0.001$ vs Dex 
aldehyde dehydrogenase 1 (ALDH1) and sex determining region $\mathrm{Y}$ (SRY)-box 2 (Sox2), was measured using qRT-PCR. The mRNA expression of ALDH1 and Sox 2 in SP cells was significantly higher than that in MP cells (Fig. 1b and d). These results showed that SP cells were successfully separated from MM cells.

\section{Cotreatment with DATS+Dex inhibited proliferation of MM SP cells and colony formation}

To investigate whether DATS+Dex affected the survival rate of MM SP cells, cells proliferation was analyzed using MTS assay as well as for colony formation and spheroid formation after drug treatment (Fig. 2). We observed that the proliferation, colony formation, and spheroid formation of SP cells were significantly lower in cells treated with DATS, Dex, or DATS+Dex than in untreated cells. Proliferation, colony formation, and spheroid formation in the DATS-treated group were lower than those in the Dex-treated group. Additionally, proliferation, colony formation, and spheroid formation following DATS+ Dex cotreatment were significantly lower than those following DATS or Dex treatment alone. These results suggested that cotreatment with DATS+Dex inhibited the proliferation and spheroid formation of
MM SP cells more effectively than either agent did alone.

\section{Cotreatment with DATS+Dex inhibited cell cycle and promoted apoptosis of SP cells}

To elucidate the effect of cotreatment with DATS+ Dex on SP cells, apoptosis and the cell cycle were evaluated after treatment with DATS, Dex, or DATS+Dex. Flow cytometry analysis revealed that the apoptosis rates following treatment with DATS, Dex, or DATS+Dex were significantly higher than those following no treatment. Apoptosis rates in the DATS group were higher than those in the Dex group, but apoptosis rate following cotreatment with DATS+Dex was much higher than that following treatment with DATS or Dex (Fig. 3a and c). In addition, the proportion of cells undergoing cell cycle arrest in the G1/S phase was altered. Compared with the untreated control group, DATS, Dex, or DATS+Dex groups showed a significantly higher percentage of cells in the G1-phase, which prevented their transition from $G 1$ to $S$ phase. The effect of DATS treatment was not significantly different from that of Dex treatment. Furthermore, DATS+Dex cotreatment markedly increased percentage of cells in the G1-phase than DATS or Dex treatment alone (Fig. $3 \mathrm{~b}$ and $\mathrm{d}$ ). These results

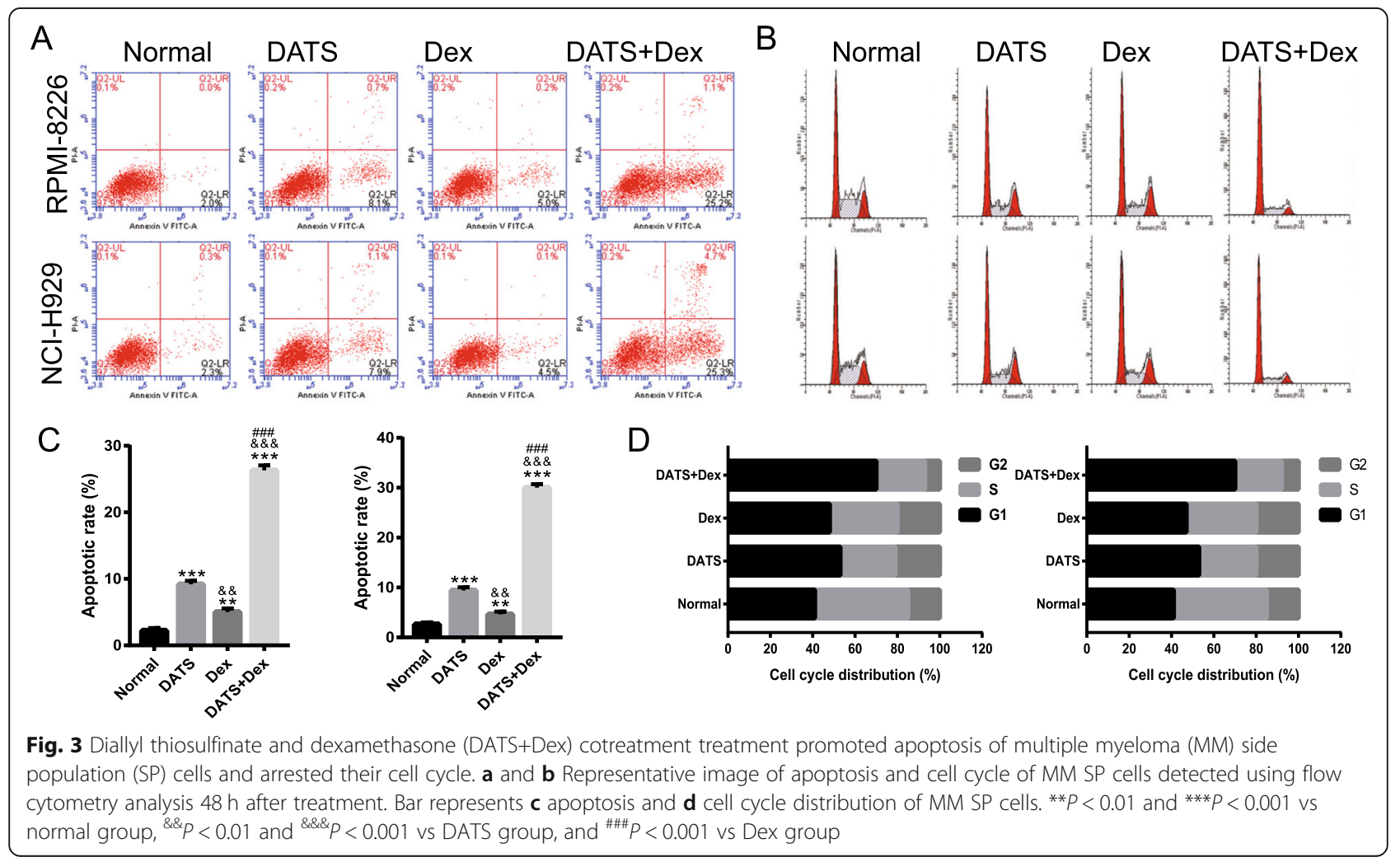


suggest that cotreatment with DATS+Dex was more effective on apoptosis and cell cycle arrest in MM SP cells than either agent alone.

\section{Cotreatment with DATS+Dex inhibited PI3K/AKT/mTOR signaling in SP cells}

We further sought to explore whether cotreatment with DATS+Dex suppressed the PI3K/AKT/mTOR signaling pathway. The expression of the components of the PI3K/AKT/mTOR pathway in SP cells was detected using western blotting after treatment with DATS, Dex, or DATS+Dex (Fig. 4). The analysis showed that the expression of PI3K, p-AKT/AKT, and $\mathrm{p}$-mTOR/mTOR was lower in MM SP cells treated with DATS, Dex, or DATS+Dex than that in untreated cells. Cotreatment with DATS+Dex clearly suppressed the protein levels of PI3K, p-AKT/AKT, and $\mathrm{p}-\mathrm{mTOR} / \mathrm{mTOR}$ more than treatment with DATS or Dex alone. Additionally, no significant differences in protein expression were observed between DATS and Dex treatments alone. These results indicated that cotreatment with DATS+Dex significantly inhibited PI3K/AKT/mTOR signaling in MM SP cells.

\section{Cotreatment with DATS+Dex promoted miR-127-3p expression in SP cells}

To explore the mechanism underlying the inhibitory effects of DATS+Dex cotreatment on the proliferation of SP cells, we further analyzed the expression of miRNAs after treatment. The results from GSE56163 showed that hsa-miR-138, hsa-miR-3200$5 p$, and hsa-miR-127-3p levels were significantly higher in SP cells than in MP cells in MM. We then measured miR-138, miR-3200-5p, and miR-127-3p expression in the SP and MP cells of RPMI-8226 and NCI-H929 cells. The results showed that miR138, miR-3200-5p, and miR-127-3p expression was significantly lower in SP cells than in MP cells

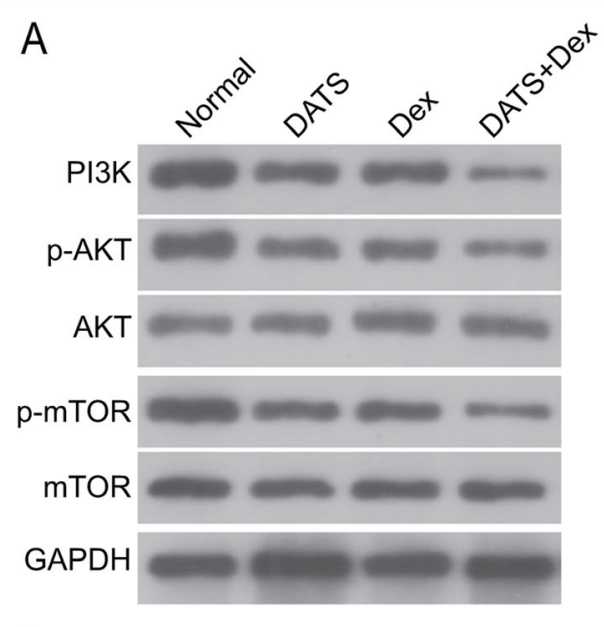

\section{$B$}
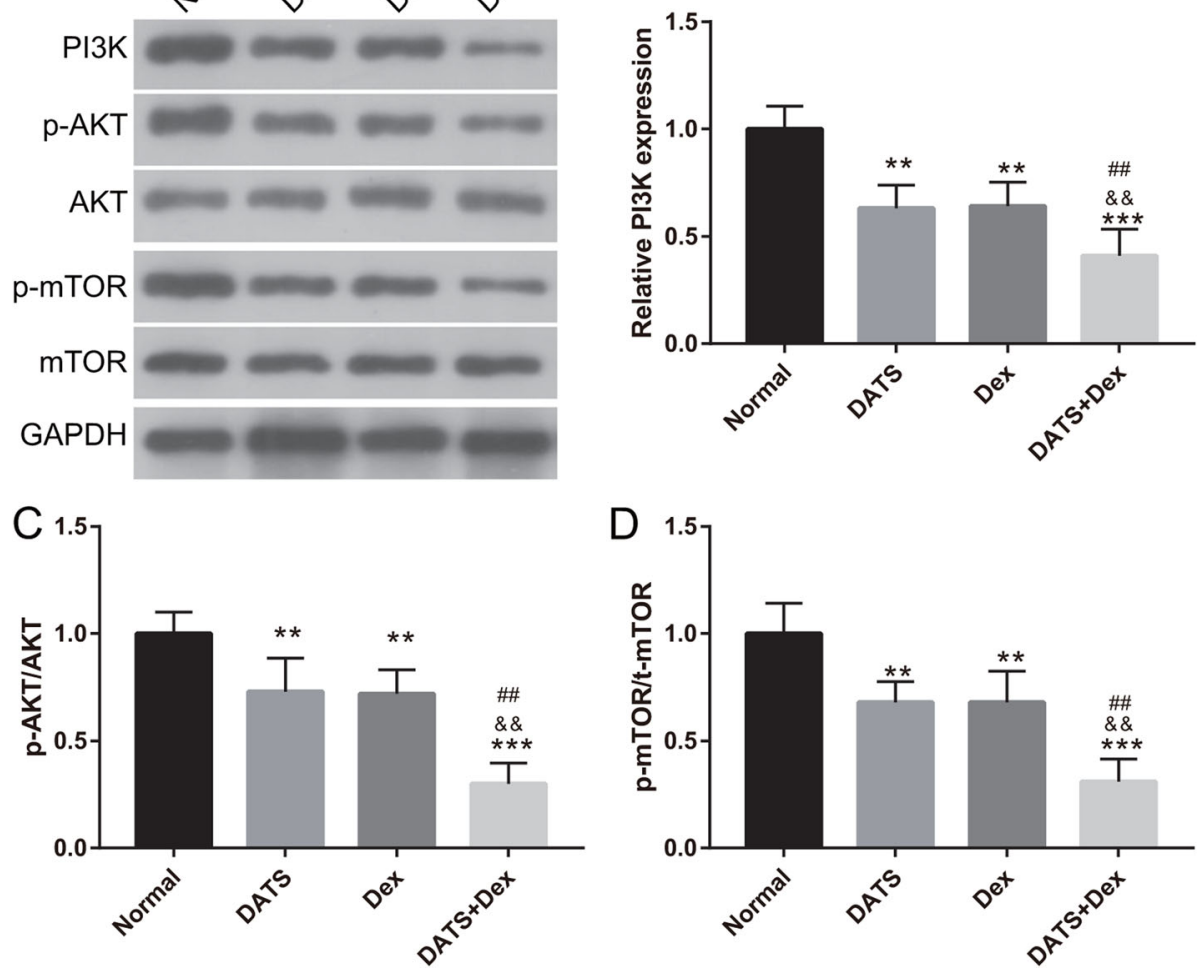

Fig. 4 Diallyl thiosulfinate and dexamethasone (DATS+Dex) cotreatment silenced phosphoinositide 3 kinase (PI3K)/AKT/mechanistic target of rapamycin (mTOR) signaling in multiple myeloma (MM) side population (SP) cells. a Expression of PI3K, AKT, mTOR, phosphorylated mTOR ( $p$ mTOR), and p-AKT was evaluated using western blotting. b-d Bar represents the expression of PI3K, p-AKT/AKT, p-mTOR/ mTOR. Note: Full-length blots gels are presented in Supplementary Figure $1{ }^{* *} P<0.01$ and ${ }^{* *} P<0.001$ vs normal group, ${ }^{\& \&} p<0.01$ vs DATS group, and ${ }^{\# \#} p<0.01$ vs Dex group 

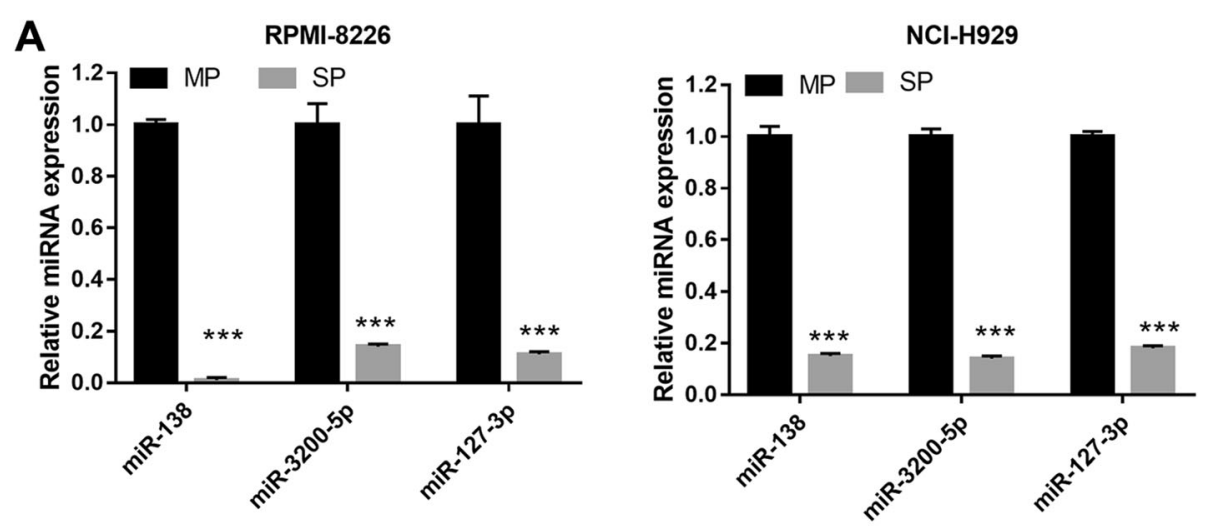

B

RPMI-8226

\section{NCl-H929}
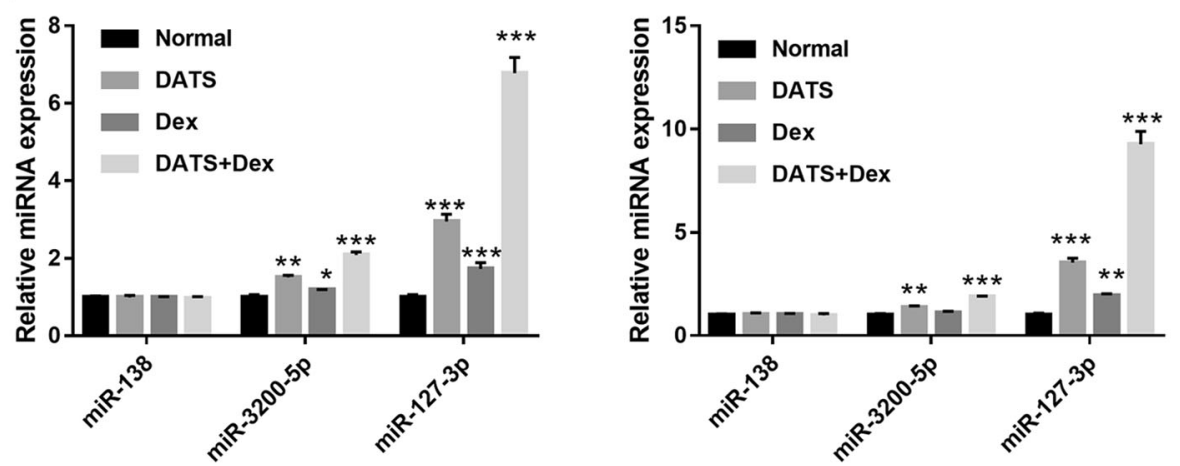

Fig. 5 Cotreatment with diallyl thiosulfinate and dexamethasone (DATS+Dex) promoted miR-3200-5p and miR-127-3p expression. a miR-138, miR-3200-5p, and miR-127-3p expression was significantly lower in side population (SP) cells than in MP cells. Expression of all miRNAs in SP and MP cells of RPMI-8226 and NCl-H929 cell lines was measured using quantitative reverse transcription-polymerase chain reaction (qRT-PCR). $\mathbf{b}$ DATS, Dex, and DATS+Dex treatment significantly increased miR-3200-5p and miR-127-3p expression in MM SP cells, but did not affect miR-138 expression, compared with no treatment. ${ }^{*} P<0.05$, ${ }^{* *} P<0.01$, and ${ }^{* * *} P<0.001$ vs normal group

(Fig. 5a). Additionally, we found that DATS, Dex, and DATS+Dex treatments induced significantly higher miR-3200-5p and miR-127-3p expression, especially of the latter, than control treatment in MM SP cells (Fig. 5). Therefore, we chose miR-127-3p for the further experiments.
Silencing of miR-127-3p expression weakened DATS+Dex cotreatment effect

Next, we explored whether cotreatment with DATS+ Dex inhibited the proliferation of SP cells by regulating miR-127-3p. First, qRT-PCR of MM SP cells transfected with an miR-127-3p inhibitor showed
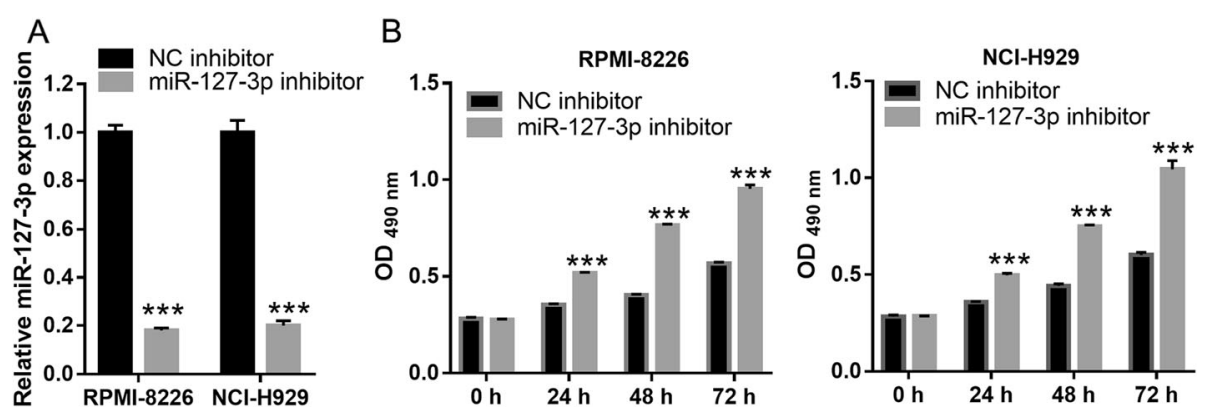

Fig. $\mathbf{6}$ Silencing miR-127-3p expression reversed effect of cotreatment with diallyl thiosulfinate and dexamethasone (DATS+Dex) on proliferation of multiple myeloma (MM) side population (SP) cells. a miR-127-3p expression was inhibited in cells transfected with miR-127-5p. b Proliferation of MM SP cells detected using MTS analysis at $0,24,48,72 \mathrm{~h}$ after treatment. Silencing miR-127-3p expression promoted proliferation of MM SP cells cotreated with DATS+Dex. ${ }^{* *} P<0.001$ 


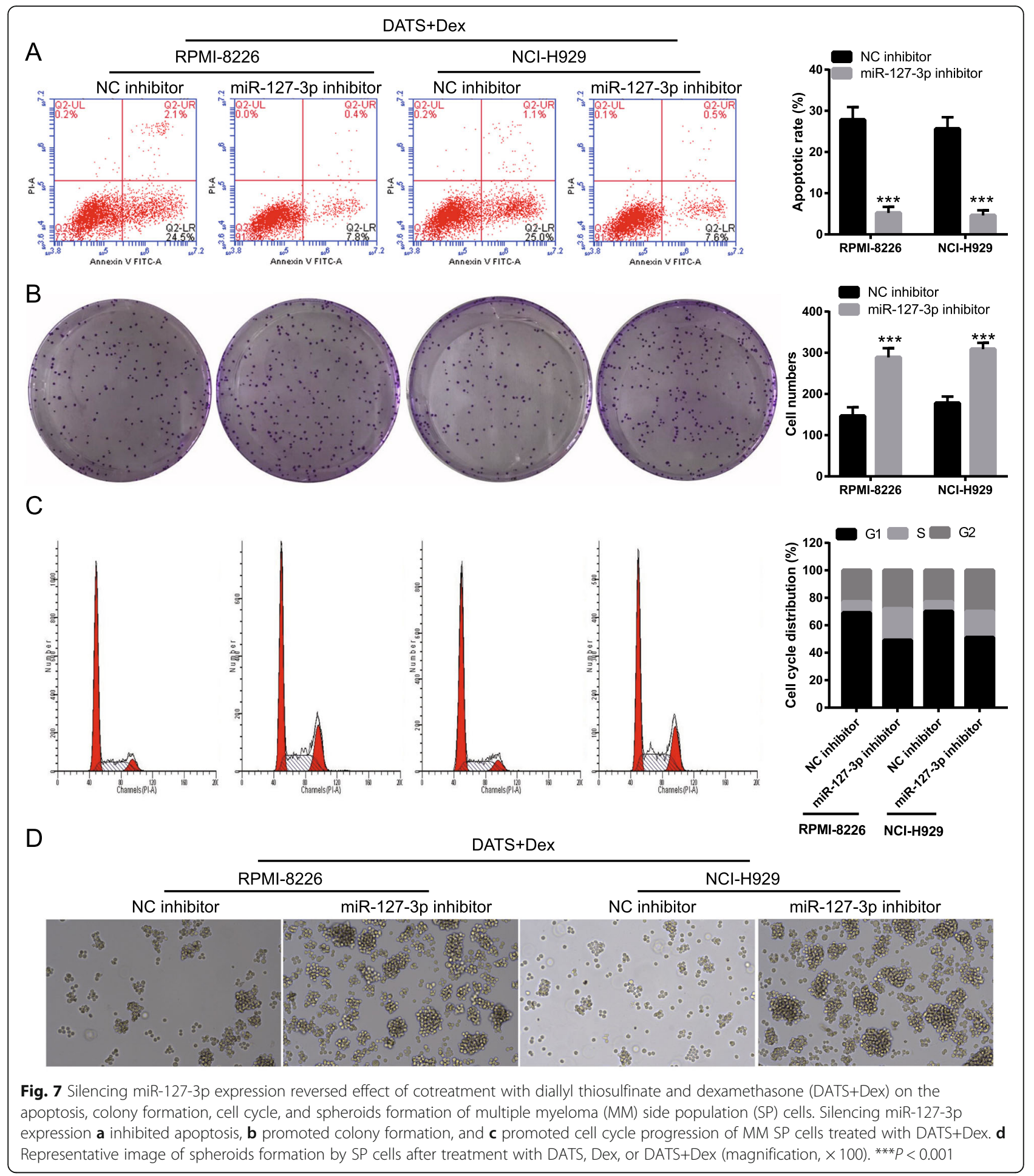

that miR-127-3p expression was inhibited after transfection (Fig. 6a). Additionally, silencing miR-127-3p significantly promoted the proliferation of MM SP cells treated with DATS+Dex (Fig. 6b). The flow cytometry assay indicated that silencing miR-127-3p significantly inhibited cell apoptosis and promoted cell cycle progression and colony formation in MM SP cells treated with DATS+Dex (Fig. 7). As shown in Fig. 8, silencing miR-127-3p increased the expression of PI3K, p-AKT/AKT, and p-mTOR/mTOR in MM SP cells treated with DATS+Dex. These results suggest that silencing miR-127-3p weakened the 


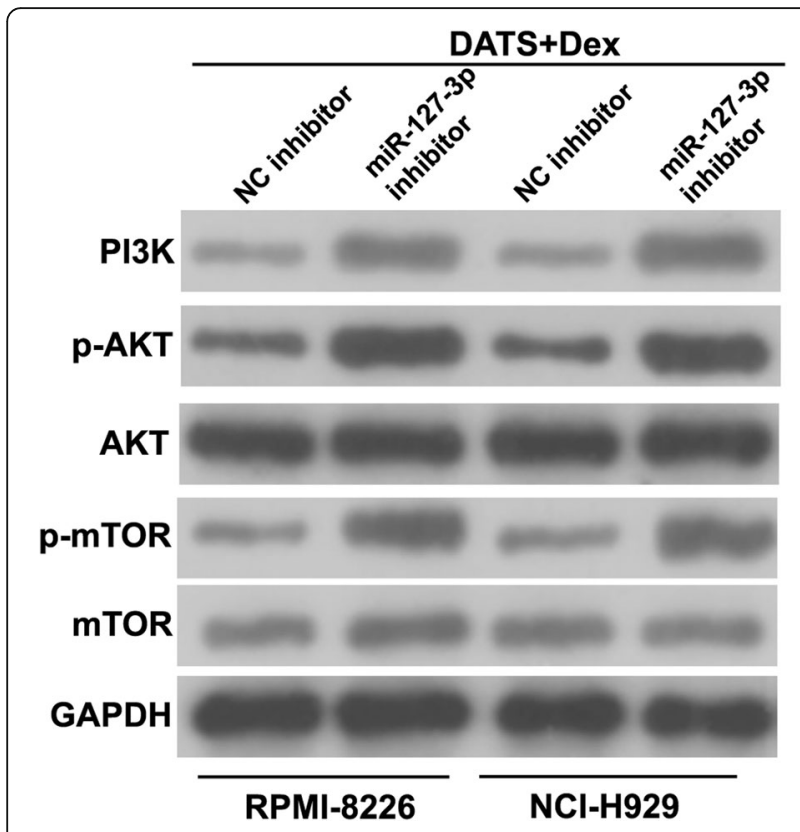

Fig. 8 Silencing miR-127-3p expression reversed effect of cotreatment with diallyl thiosulfinate and dexamethasone (DATS+Dex) on phosphoinositide 3-kinase (PI3K) pathway of multiple myeloma (MM) side population (SP) cells. Expression of PI3K, phosphorylated AKT (p-AKT)/AKT, and p-mechanistic target of rapamycin (mTOR)/mTOR in MM SP cells analyzed using western blotting. Note: Full-length blots gels are presented in Supplementary Figure 2

effect of DATS+Dex cotreatment on the proliferation of SP cells.

\section{Discussion}

DATS is a herbal drug that has long been known for its medicinal properties [19], including antitumor activity [7, 8]. Gao et al. [20] reported that DATS enhances the efficiency of cyclophosphamide in the treatment of neuroblastoma. Wang et al. [21] have reported that recombinant interleukin-2 plus DATS cotreatment suppresses pancreatic cancer. In our previous study, we found that DATS inhibited the proliferation and colony formation of MM SP cells, inducing G1/S arrest [9]. In this study, we found that compared with DATS or Dex treatment alone, cotreatment with DATS+Dex resulted in greater inhibition of colony formation, spheroids formation, and proliferation of SP cells and in greater induction of apoptosis and cell cycle arrest in the G1/S phase. These results suggest that cotreatment with DATS+Dex inhibited proliferation and promoted apoptosis of MM SP cells more effectively than treatment with DATS or Dex alone.

miRNAs are emerging as important modulators of cellular signaling, including cell proliferation, in MM. In this study, we found that miR-127-3p expression was more significantly inhibited in MM SP cells than it was in MM MP cells. A previous study found that miR-127-3p expression was significantly reduced and that the miRNA acted as tumor suppressors in gastric, prostatic, and ovarian cancers [22-24]. In this study, we found that treatment with DATS and Dex alone or in combination, significantly promoted miR127-3p expression and that silencing miR-127-3p reversed the effect of these treatments on the proliferation of SP cells. These results suggest that miR-127$3 p$ acts as a tumor suppressor in MM, similar to that in other cancers. DATS+Dex cotreatment inhibited the proliferation of MM SP cells by upregulating miR-127-3p expression.

The PI3K/AKT/mTOR signaling pathway is known to control cell survival and is abnormally activated in a wide variety of cancers, resulting in inhibition of apoptosis via multiple mechanisms [25]. Recent studies have shown that the activation of the PI3K/AKT/mTOR signaling pathway plays a key role in the survival and proliferation of MM cells [26, 27]. In the present study, a combination of DATS+Dex decreased the expression of PI3K, p-AKT/AKT, and p-mTOR/mTOR, suggesting that DATS+Dex cotreatment deactivated the PI3K/AKT signaling pathway. Additionally, silencing miR-127-3p activated the PI3K/AKT signaling pathway and reversed the effect of DATS and Dex treatment alone or in combination on the PI3K/AKT signaling pathway. These results showed that cotreatment with DATS+Dex negatively regulated the PI3K/AKT/mTOR signaling pathway to exert anticancer effects in MM SP cells through upregulating miR-127-3p expression.

\section{Conclusion}

Cotreatment with DATS+Dex inhibited the proliferation of MM SP cells by promoting miR-127-3p expression and deactivating the PI3K/AKT signaling pathway. Our data provide a theoretical basis for the clinical application of DATS and Dex in MM patients. However, further research is needed to investigate these effects in animal models and clinical trials.

\section{Supplementary Information}

The online version contains supplementary material available at https://doi. org/10.1186/s12885-021-07833-5.

\section{Additional file 1.}

Additional file 2.

\section{Abbreviations}

MM: Multiple myeloma; SP: Side population; MP: Main population; Dex: Dexamethasone; DATS: Diallyl thiosulfinate; miRNAs: MicroRNAs

Acknowledgements

None. 


\section{Authors' contributions}

$\mathrm{WH}$ and YF made substantial contributions to the conception and design of the work, the acquisition and analysis of data, and drafted the manuscript. WH, YF, and YZ have made substantial contributions to the acquisition and analysis of data. XW and BL interpreted the data. JZ made substantial contributions to the conception and design of the work and revised the manuscript. All authors have read and approved the final manuscript.

\section{Funding}

This study was supported by the Jiangxi Educational Department Foundation of China (No. GJJ170628), Science and Technology Program of Jiangxi Administration of Traditional Chinese Medicine (2019A178), and the Nature Science Foundation of Jiangxi Province (20181BAB205024 and 20202BABL206161). The funders played no role in the design, execution, analysis, interpretation, or presentation of this work.

\section{Availability of data and materials}

The datasets used, analyzed, or both during the current study are available from the corresponding author upon reasonable request.

\section{Ethics approval and consent to participate}

Not applicable.

\section{Consent for publication}

Not applicable.

\section{Competing interests}

The authors declare that they have no competing interests.

\begin{abstract}
Author details
'Jiangxi Key Laboratory of Molecular Medicine, The Second Affiliated Hospital of Nanchang University, Nanchang 330006, Jiangxi Province, China. ${ }^{2}$ Department of Psychiatry, Jiangxi Mental Hospital, Nanchang 330029, Jiangxi Province, China. ${ }^{3}$ Department of Hematology, The Affiliated Hospital of Jinggangshan University, ji'an 343000, Jiangxi Province, China. ${ }^{4}$ Comprehensive teaching and research office, Ji'an College, ji'an 343000, Jiangxi Province, China. ${ }^{5}$ Department of internal medicine, Jinggangshan University, ji'an 343009, Jiangxi Province, China.
\end{abstract}

Received: 21 June 2020 Accepted: 11 December 2020 Published online: 06 February 2021

\section{References}

1. Mahindra A, Hideshima T, Anderson KC. Multiple myeloma: biology of the disease. Blood Rev. 2010;24(Suppl 1):S5-11.

2. Fayaz SM, Suvanish Kumar VS, Rajanikant GK. Necroptosis: who knew there were so many interesting ways to die? CNS Neurol Disord Drug Targets. 2014;13(1):42-51.

3. Goodell MA, Brose K, Paradis G, Conner AS, Mulligan RC. Isolation and functional properties of murine hematopoietic stem cells that are replicating in vivo. J Exp Med. 1996;183(4):1797-806.

4. Wang L. Li Y: [research advances of side population cells in multiple myeloma -review]. Zhongguo shi yan xue ye xue za zhi. 2017;25(4):1250-4

5. Raab MS, Podar K, Breitkreutz I, Richardson PG, Anderson KC. Multiple myeloma. Lancet. 2009;374(9686):324-39.

6. Yan W, Du J, Du Y, Pu H, Liu S, He J, Zhang J, Hou J. Fenretinide targets the side population in myeloma cell line $\mathrm{NCl}-\mathrm{H} 929$ and potentiates the efficacy of antimyeloma with bortezomib and dexamethasone regimen. Leuk Res. 2016;51:32-40

7. Mikaili P, Maadirad S, Moloudizargari M, Aghajanshakeri S, Sarahroodi S. Therapeutic uses and pharmacological properties of garlic, shallot, and their biologically active compounds. Iran J Basic Med Sci. 2013;16(10):1031-48.

8. Lai KC, Hsu SC, Yang JS, Yu CC, Lein JC, Chung JG. Diallyl trisulfide inhibits migration, invasion and angiogenesis of human colon cancer HT-29 cells and umbilical vein endothelial cells, and suppresses murine xenograft tumour growth. J Cell Mol Med. 2015;19(2):474-84.

9. Zeng JQ, Zheng YL, Liu B, Lian JP, Gong ZN. Mechanism of DATS inhibiting side population cell proliferation in multiple myeloma. Zhongguo Shi Yan Xue Ye Xue Za Zhi. 2017;25(4):1086-91.
10. Wang H, Sun N, Li X, Li K, Tian J, Li J. Diallyl trisulfide induces osteosarcoma cell apoptosis through reactive oxygen species-mediated downregulation of the PI3K/Akt pathway. Oncol Rep. 2016;35(6):3648-58.

11. Shin DY, Kim GY, Hwang HJ, Kim WJ, Choi YH. Diallyl trisulfide-induced apoptosis of bladder cancer cells is caspase-dependent and regulated by PI3K/Akt and JNK pathways. Environ Toxicol Pharmacol. 2014;37(1): 74-83.

12. Niu NK, Wang ZL, Pan ST, Ding HQ, Au GH, He ZX, Zhou ZW, Xiao G, Yang $Y X$, Zhang $X$, et al. Pro-apoptotic and pro-autophagic effects of the Aurora kinase a inhibitor alisertib (MLN8237) on human osteosarcoma U-2 OS and MG-63 cells through the activation of mitochondria-mediated pathway and inhibition of p38 MAPK/PI3K/Akt/mTOR signaling pathway. Drug Design Dev Therapy. 2015;9:1555-84.

13. Xu P, Xia T, Ling Y, Chen B. MiRNAs with prognostic significance in multiple myeloma: a systemic review and meta-analysis. Medicine. 2019;98(33): e16711.

14. Huang YH, Chen ST, Liu FH, Hsieh SH, Lin CH, Liou MJ, Wang CC, Huang CH, Liu GH, Lin JR, et al. The efficacy and safety of concentrated herbal extract granules, YH1, as an add-on medication in poorly controlled type 2 diabetes: a randomized, double-blind, placebo-controlled pilot trial. PLoS One. 2019;14(8):e0221199.

15. Wu H, Liu C, Yang Q, Xin C, Du J, Sun F, Zhou L. MIR145-3p promotes autophagy and enhances bortezomib sensitivity in multiple myeloma by targeting HDAC4. Autophagy. 2020;16(4):683-97.

16. Yuan X, Ma R, Yang S, Jiang L, Wang Z, Zhu Z, Li H. miR-520g and miR-520h overcome bortezomib resistance in multiple myeloma via suppressing APE1. Cell Cycle (Georgetown, Tex). 2019;18(14):1660-9.

17. Du J, Liu S, He J, Liu X, Qu Y, Yan W, Fan J, Li R, Xi H, Fu W, et al. MicroRNA451 regulates stemness of side population cells via PI3K/Akt/mTOR signaling pathway in multiple myeloma. Oncotarget. 2015;6(17):14993-5007.

18. Zeng J, Liu T, Zheng Y, Liu B, Fang M, Long S, Chen Y. Diallyl trisulfide inhibits proliferation and promotes apoptosis of side population cells in multiple myeloma cells, vol. 10; 2017.

19. Petrovska BB, Cekovska S. Extracts from the history and medical properties of garlic. Pharmacogn Rev. 2010;4(7):106-10.

20. Gao XY, Geng XJ, Zhai WL, Zhang XW, Wei Y, Hou GJ. Effect of combined treatment with cyclophosphamidum and allicin on neuroblastoma-bearing mice. Asian Pac J Trop Med. 2015;8(2):137-41.

21. Wang CJ, Wang C, Han J, Wang YK, Tang L, Shen DW, Zhao Y, Xu RH, Zhang $\mathrm{H}$. Effect of combined treatment with recombinant interleukin-2 and allicin on pancreatic cancer. Mol Biol Rep. 2013;40(12):6579-85.

22. Guo LH, Li H, Wang F, Yu J, He JS. The tumor suppressor roles of miR-433 and miR-127 in gastric Cancer. Int J Mol Sci. 2013;14(7):14171-84.

23. Fan J, Du W, Zhang H, Wang Y, Li K, Meng Y, Wang J. Transcriptional downregulation of miR-127-3p by CTCF promotes prostate cancer bone metastasis by targeting PSMB5. FEBS Lett. 2020;594(3):466-76.

24. Liu X, Meng Z, Xing Y, Zhong Q, Zhang X, Qu J. MiR-127 inhibits ovarian cancer migration and invasion by up-regulating ITGA6. Minerva Med. 2019. https://doi.org/10.23736/S0026-4806.19.06237-2. Online ahead of print.

25. Vivanco I, Sawyers CL. The phosphatidylinositol 3-kinase AKT pathway in human cancer. Nat Rev Cancer. 2002;2(7):489-501.

26. Hsu J, Shi Y, Krajewski S, Renner S, Fisher M, Reed JC, Franke TF, Lichtenstein A. The AKT kinase is activated in multiple myeloma tumor cells. Blood. 2001; 98(9):2853-5.

27. Pene F, Claessens YE, Muller O, Viguie F, Mayeux P, Dreyfus F, Lacombe C, Bouscary D. Role of the phosphatidylinositol 3-kinase/Akt and mTOR/P70S6kinase pathways in the proliferation and apoptosis in multiple myeloma. Oncogene. 2002;21(43):6587-97.

\section{Publisher's Note}

Springer Nature remains neutral with regard to jurisdictional claims in published maps and institutional affiliations. 